\title{
Adsorption and degradation mechanisms of perfluoroalkyl substances (PFAS) on Ag- exchanged FAU-type zeolite studied by in situ synchrotron X-ray diffraction and thermal analysis/isotope ratio mass spectrometry.
}

\author{
M. Mancinelli ${ }^{1}$, L. Gigli ${ }^{2}$, M. Ardit ${ }^{1}$, J.R. Plaisier ${ }^{2}$, G. Bianchini ${ }^{1}$, G. Salani ${ }^{1}$, A. Martucci ${ }^{1}$ \\ ${ }^{1}$ Department of Physics and Earth Sciences, University of Ferrara Via Saragat 1, I-44121, Ferrara, Italy. \\ ${ }^{2}$ Elettra-Sincrotrone Trieste S.C.p.A., Materials Characterisation By X-ray diffraction (MCX) Beamline, Strada Statale 14 - $\mathrm{km} 163,5$ \\ in AREA Science Park, Basovizza, Trieste, Italy. \\ maura.mancinelli@unife.it
}

In recent years, the occurrence and fate of perfluoroalkyl substances (PFAS) in the aquatic environment was recognized as one of the emerging issues in environmental chemistry. In particular, PFOA (C8HF15O2) and PFOS (C8HF17O3S) have recently become the targets of global concern due to their ubiquitous presence in the environment, persistence, and bioaccumulative properties. Strong carbon-fluorine (C-F) bonds make PFOA and PFOS extremely resistant to chemical, thermal and biological degradation, consequently, their removal from water is a crucial scientific and social challenge [1-2]. This work aims to investigate, for the first time, the structural modifications and the desorption kinetics during the thermal activation of FAU and Ag-exchanged FAU-type zeolites used for removal of PFOA and PFOS from water. The introduction of Ag in the framework confers them unique physical, chemical, and antibacterial properties along with strong absorption property, good stability and catalytic activity [3]. In situ hightemperature synchrotron X-ray powder diffraction and thermal analysis coupled with Elemental Analyzer - Isotope Ratio Mass Spectrometry (EA-IRMS) provided to: i) investigate high temperature structural modifications experienced after during PFOA and PFOS thermal desorption and to check the crystallinity preservation of the porous matrix; ii) monitor and evaluate the organics decomposition process upon heating; iii) estimate the influence of $\mathrm{Ag}$ active sites in working zeolites; iv) verify the best regeneration temperature in order to verify whether they can be re-used for PFAS removal from wastewater. This information was crucial for designing and optimizing the regeneration treatment of such zeolites, which are revealed to be highly effective in water-remediation technology. Powder diffraction data of each sample were collected at the synchrotron MCX beamline (Elettra, Trieste, Italy) and were subjected to the same heat treatment profile encompassing the heating ramp with a rate of $5^{\circ} \mathrm{C} / \mathrm{min}$ from room temperature to $800{ }^{\circ} \mathrm{C}$. The powder samples were loaded and packed into a $0.5 \mathrm{~mm}$ quartz capillary open at one end and heated in situ using a hot air stream. The analysis of the patterns collected using in situ synchrotron XRPD on zeolites showed no heat-induced symmetry change. Moreover, to carry out a comparison, structural data relating to bare zeolites are also reported in order to check the contribution of the loaded organic molecules. The differential thermal analysis (DTA) curve shows two DTA exothermic events between 300 and $600{ }^{\circ} \mathrm{C}$ due to the PFAs degradation, in very good agreement with the EA-IRMS analyses. The complete thermal regeneration of selected materials was achieved at $\sim 600$ and $750^{\circ} \mathrm{C}$ for PFOA and PFOS loaded samples, respectively. No relevant variations were observed in Ag-exchanged materials. This information is crucial to examine the efficiency of Ag-exchanged zeolites compared to same samples taken separately for the adsorption of PFOS and PFOA from water. The diffraction peaks were also accurately monitored to study in real-time their evolution (in terms of broadening, shift, changes of intensities) compared with the $\mathrm{Y}$ zeolites without any treatment, after silver activation and as well as after PFOS and PFOA adsorption. The XRD analysis demonstrated that the adsorption/desorption process occurred without significant loss of zeolite crystallinity, but with slight deformations in the channel apertures. The regenerated zeolites regain the unit-cell parameters of the bare materials almost perfectly, however. Only a slight memory effect in terms of structural deformations is registered in channel geometry thus demonstrating that the regenerated samples which could be able to readsorb similar amounts of PFOS.

[1] Ferreira, L., Fonseca, A.M., Botelho, G, Almeida-Aguiar, C, Neves, I.C. (2012). Antimicrobial activity of faujasite zeolites doped with silver. Microporous and Mesoporous Materials. 160, 126-132.

[2] Kucharzyk, K.H., Darlington, R., Benotti, M., Deeb, R., Hawley, E. (2017). Novel treatment technologies for PFAS compounds: A critical review. Journal of Environmental Management. 204, 757-764.

[3] Ziwen, D., Denga, S., Bei, Y., Huang, Q., Wang, B., Huang, J., Yua, G. (2014). Adsorption behavior and mechanism of perfluorinated compounds on various adsorbents-A review. Journal of Hazardous Materials. 274, 443-454.

Keywords: perfluoroalkyl substances, emerging contaminants, in situ-synchrotron X-ray diffraction, EA-IRMS, zeolites. 